\title{
Past, Present and Future of Chinese Astronomy
}

\author{
Cheng Fang \\ School of Astronomy and Space Science, Nanjing University, 22 Han Kou road, Nanjing, China \\ email: fangc@nju.edu.cn
}

\begin{abstract}
Through out the ancient history, Chinese astronomers had made tremendous achievements. Since the main purpose of the ancient Chinese astronomy was to study the correlation between man and the universe, all the Emperors made ancient Chinese astronomy the highly regarded science throughout the history. After a brief introduction of the achievement of ancient Chinese astronomy, I describe the beginnings of modern astronomy research in China in the 20th century. Benefiting from the fast development of Chinese economy, the research in astronomy in China has made remarkable progress in recent years. The number of astronomers has doubled in the past ten years, and the number of graduate students has grown over 1300. The current budget for astronomy research is ten times larger than that ten years ago. The research covers all fields in astronomy, from galaxies to the Sun. The recent progress in both the instruments, such as the Guo Shoujing's telescope, a Large Sky Area Multi-Object Fiber Spectroscopic Telescope (LAMOST), and the theoretical research will be briefly presented. The ongoing and future projects on the space- and ground-based facilities will be described, including the Five Hundred Meter Aperture Spherical Radio Telescope (FAST), "Chang E" (Lunar mission) project, Hard X-ray Modulate Telescope (HXMT), DArk Matter Particle Explorer (DAMPE), Deep Space Solar Observatory (DSO), Chinese Antarctic Observatory (CAO), 65m steerable radio telescope, Chinese Spectral Radioheliogaph $(\mathrm{CSRH})$ etc.
\end{abstract}

Keywords. history, ancient astronomy, present status, future astronomy, China

Through out the ancient history, Chinese astronomers had made tremendous achievements. They maintained the longest continuous records of all kinds of astronomical phenomena, which are still useful today for astronomical research. They invented various important astronomical instruments for astronomical observations and measurements. However, during the 18-19th century Chinese astronomy fell behind much due to the feudal and introverted Emperor's control.

\section{Ancient Astronomy in China}

In ancient times, the Emperors of various Dynasties nominated some special officers to observe stars. For instance, a legend said that astronomers Xi and He received commissions from Emperor Yao to observe stars and to make calendars. Figure 1 shows an ancient nomination ceremony before the Emperor.

There were mainly two reasons for observing stars: (1) The astrological and political reasons. Eclipse observations are good examples. People used to believe that during the eclipses, the Sun was devored by a celestial dog, an omen portending all kinds of disasters. So the Emperors and people would like to know when and how the eclipse would appear. (2) To make good astronomical systems. For instance, every dynasty needed astronomical chronology and made the calendars, etc.

For a period of over 3000 years, Chinese made tremendous progress in astronomy. They maintained the longest continuous records of all kinds of astronomical phenomena, 


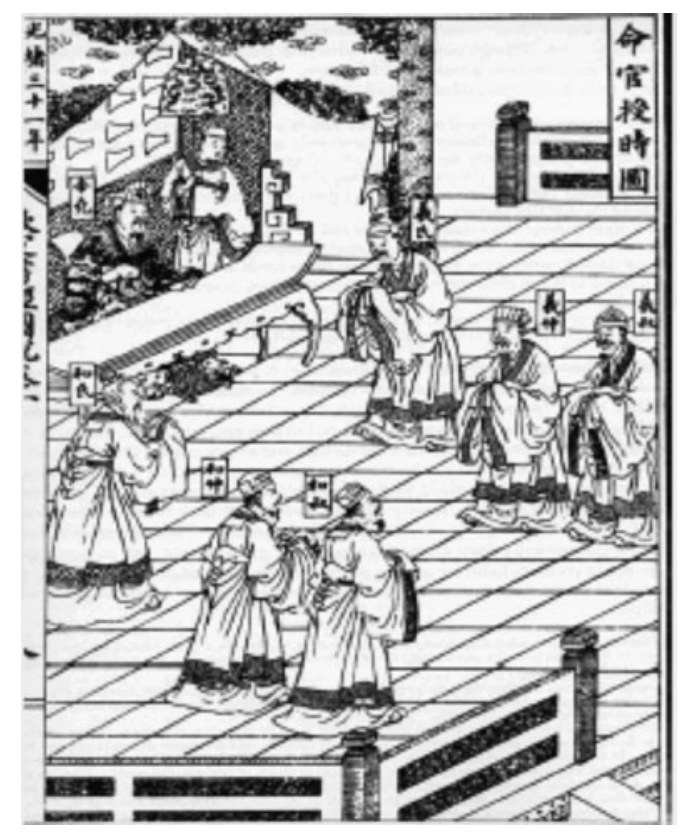

Figure 1. Ancient Chinese astronomers were nominated by Emperors.

which are still useful today for astronomical research. Ancient Chinese astronomers made more than 100 astronomical calendars, each being a sophisticated system of astronomical computation, more than 50 of them were officially used; They also constructed a large number of astronomical instruments. The construction of the Astronomical Clock Tower in the 11th century is a great example.

Ancient Chinese kept records of a wide range of celestial events, including solar and lunar eclipses, sunspots, comets and meteors, guest stars (nova or supernova), planetary events, aurora borealis etc.

\subsection{Historical eclipse records in China}

Solar eclipses have already been mentioned in oracle bone inscriptions since Shang Dynasty (1600 B.C-1046 B.C), as seen in the left panel in Figure 2. For instance, some records on oracle bones indicate that solar eclipses occurred on October 21, 1198 B.C, June 7, 1172 B.C, and October 31, 1161 B.C. etc. Generally the recorded data include the position of the Sun at the time of eclipse, the beginning and the end of the eclipse, the magnitude of the eclipse, the point of the first contact on the solar disc etc.

The ancient records of solar eclipses have been used to study the secular change of the Earth's rotation. A decreasing rotation rate has been found to be $-22--26$ seconds/century. The records can also be used for astronomical chronology. For example, the Bamboo Chronicles says: "During the first year of King Yi of the Zhou Dynasty, two daybreaks occurred.". According to the eclipse that occurred in 899 B.C, people can tell exactly the day of the year. By using these records, people have discovered the periodicity of eclipses. For instance, ancient Chinese already knew the 135 synodic months in 100 B.C. In $762 \mathrm{AD}$, a period of 458 months was discovered, which was confirmed by Newcomb 1100 years later. In 1199 AD, the Saros Cycle (223 months) was discovered. 




Figure 2. Solar eclipses recorded in oracle bones (left) and sunspot recorded as a gold bird (right).
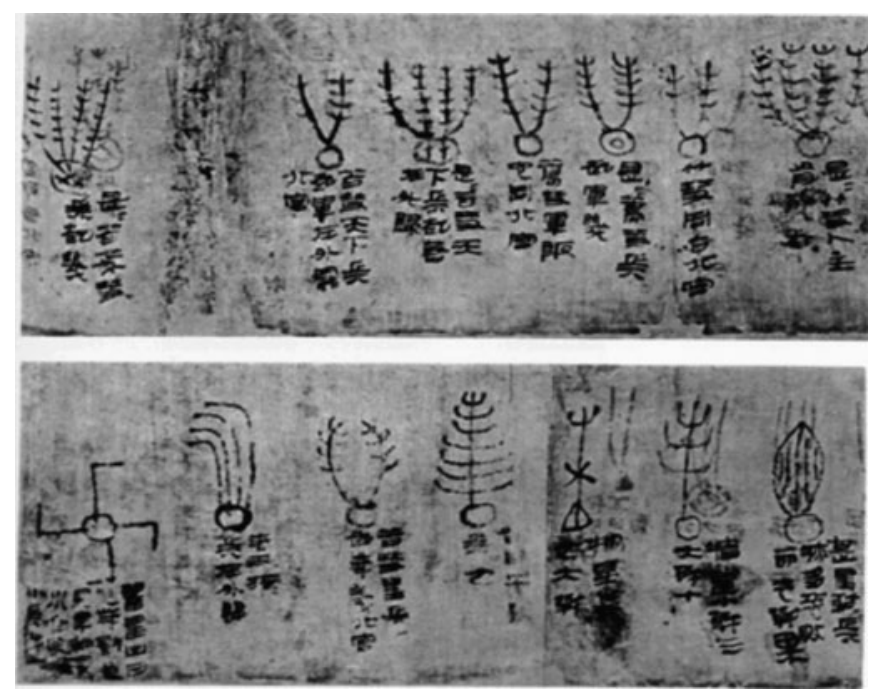

Figure 3. The silk book from the Former Han (206 B.C-8 AD) had pictures of all types of comets.

\subsection{Ancient sunspot records}

In fact, sunspots had been recorded in the book of I Ching about around 800 B.C. The first well-recognized sunspot record was made back in 28 AD, Han Dynasty, describing the sunspot as "dark air like coin on the Sun". From Han to Ming Dynasty there were more than 100 sunspot records (Xu \& Jiang 1986). Sunspots were depicted "like bird", "like coin" or "like chestnut", while the records of "disappeared in several months" and "disappeared in three days" indicate the evolution of sunspots. During Han Dynasty, there was a record of sunspot as a "Gold bird" (right penal in Fig. 2), which was discovered in a famous tomb in Hunan province.

\subsection{Pictures of all types of comets}

The silk book from the Former Han (206 B.C-8 AD) had pictures of all types of comets (Fig. 3). The shapes and states of the comets were meticulously represented.

\subsection{Ancient astronomical instruments}

The reason that ancient Chinese could made such great advancements in calendric astronomy was that they invented various important astronomical instruments for astronomical 

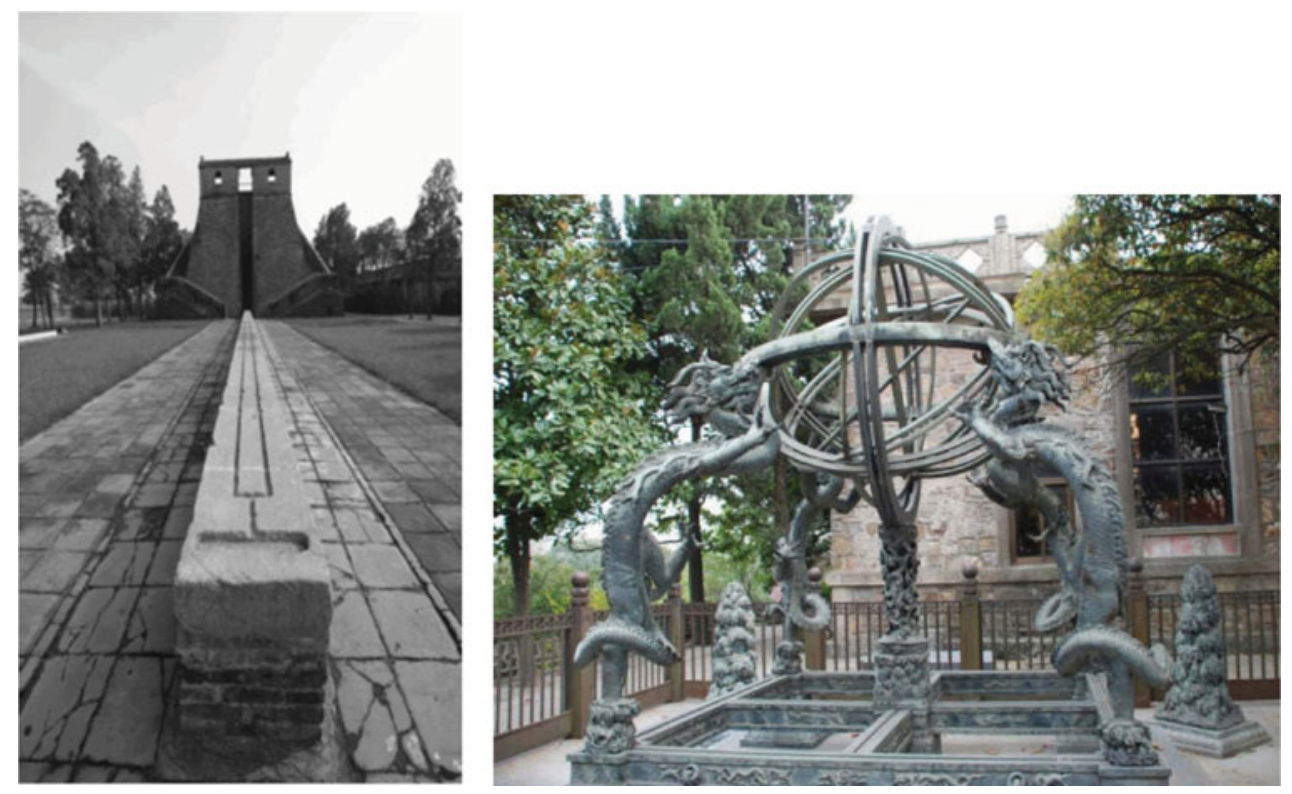

Figure 4. A large gnomon in the beginning of the Yuan Dynasty (left) and the Armillary Sphere, a replica of Guo Shoujings instrument in 1442 (right).

observations and measurements. Among them, the Gnomon is perhaps the oldest astronomical instrument of ancient China. By measuring the gnomon shadow length at noon, one can tell the season of the year. To increase the accuracy of gnomon measurements, Guo Shoujing (1231-1316) constructed a large gnomon in the beginning of the Yuan Dynasty (left penal in Fig. 4).

The right penal in Figure 4 shows the Armillary Sphere, a replica of Guo Shoujings instrument in 1442. The positioning of the rings can be adjusted to reflect such celestial movements as precession and the retrograde of the nodes of the lunar path and the ecliptic. Another instrument made also by Guo Shoujing is the simplified Instrument, Jian yi.

The making of Chinese astronomical instrument reached a peak in the 11th century, during the North Song Dynasty, when Su Song (1020-1101) and his team constructed the water powered astronomical clock tower. It was an automated mechanical system powered by water. An escapement mechanism was used to control the movement of the driving wheel. Joseph Needham hailed it as the first astronomical clock in the world.

\section{Beginnings of Modern Astronomy Research and Present Status in China}

Several events marked the beginnings of modern astronomy in China: On 1922 October 30, more than 40 scholars held a meeting at the Beijing Ancient Astronomical Observatory to found the Chinese Astronomical Society; The Purple Mountain Observatory (PMO), the biggest observatory in East Asia at that time, was built in 1934; One year later, the fifth General Assembly of the international Astronomical Union (IAU) was held in Paris, and China became a member of the IAU .

Since the establishment of the People's Republic of China, and in particular, after the so-called great cultural revolution, Chinese astronomy has advanced quickly. People 
realized that the international collaboration was important for developing astronomy in China. Thus the first Chinese astronomer delegation visited Kitt Peak Observatory of the USA in 1976. In succession, the first USA astronomer delegation visited PMO in 1977. Six years later, the first international workshop on solar physics was successfully organized in Kunming in 1983.

Benefiting from the fast development of Chinese economy, the research of astronomy in China has achieved remarkable progress in recent years. The Chinese Astronomical Society now has 2481 members. There are about 400 researchers and professors, twice more than that ten years ago, and 1300 graduate students. The main astronomical organizations include Purple Mountain Observatory (PMO), National Astronomical Observatories of China (NAOC, Beijing), Yunnan Astronomical Observatory (YAO, NAOC), Xinjiang Astronomical Observatory (XAO, NAOC), Nanjing Astronomical Instrument Research Center (NAIRC, NAOC), Shanghai Astronomical Observatory (SAO), and National Time Service Center (NTSC).

In recent years, more than 20 universities have established astronomy education and research group. A complete series of educational programs of undergraduate, master, doctoral and post-doctoral levels have been set up. There are astronomy departments in five key universities, including Nanjing University (NJU, established in 1952), Beijing University (BJU, 1960), Beijing Normal University (BNU, 1960), University of Science and Technology of China (USTC, 1978), and Xiameng University at Fuzhou (2012).

Recently, Chinese astronomers have gotten many important results. Here only are some examples.

In the field of cosmology, Yipeng Jing and his group in SAO carried out a series of the state-of-the art N-body simulations of structure formation in the Universe, and obtained a number of important conclusions. Jing \& Suto (2002) studied non-spherical modeling of dark matter halos in detail, and obtained a series of practically useful fitting formulae in applying the triaxial model. Zhao et al. (2009) found universal laws governing mass growth and structure evolution of dark matter halos. Their model is much more accurate than all previous works. By analyzing the shower development in the Advanced Thin Ionization Calorimeter (ATIC) instrument, which can separate electrons from large cosmic ray background, after four flights from 2000, the cosmic ray electron spectrum has shown an excess above $100 \mathrm{GeV}$ compared to theoretical models. The excess of cosmic ray electrons is mysterious. It could be from near astrophysical objects or other exotic sources such as dark matter particles. Jin Chang and his group played an important role for these observations (Chang et al. 2006). Chinese astronomers have also developed evolutionary population synthesis and spectral fitting codes, which have been used to analyze the spectral parameters of galaxies.

In the field of high energy astrophysics, some authors proposed an universal light curves of GRB afterglows through analyzing the observational data of Swift satellite. A new direction of GRB cosmology was proposed and constrained the cosmological parameters and the properties of dark energy jointly with other cosmological probes (Dai et al. 2004). In the aspect of AGNs, a theory of advection-dominated accretion flow disk of black holes was developed and applied to the center black hole of the Galaxy. It can explain the observations successfully. Chinese scientists derived the magnetic field of some neutron stars through the "propeller" effect, suggested a method of direct measurement of the mass of black holes and neutron stars by observing the Doppler-shifted absorption lines from accretion disk winds.

In the field of the Galaxy research, using all HII regions and giant molecular clouds, Jinlin Han and his group derived the spiral arm structure, which is probably the best known structure of our Milky Way (Hou et al. 2009). They used pulsar rotation 
measurement to obtain the magnetic fields in the Galactic disk (Han et al. 2006) and used the rotation measure sky to deduce the magnetic fields in the Galactic halo (Han et al. 1999). Using VLBA and some maser sources in the Milky Way, Xingwu Zheng and his group made first parallax measurement and deduced the distances and the proper motions of the sources with the accuracy as high as 0.05 mas, so it provides the possibility to map the spiral structure of the Milky Way (Xu et al. 2006). By measuring the parallax and proper motions of high mass star forming regions, they found that the Milky Way was rotating about $15 \%$ faster than previously estimated (Xu et al. 2009). This implies that the mass of the Milky Way's dark matter halo would be increased by $50 \%$.

In the field of stellar physics, a group led by Gang Zhao in the NAOC has gotten a batch of valuable results on stellar chemical abundance, especially in metal-poor stars (e.g. Zhao \& Gehren 2000). Zhanwen Han et al. (e.g. Han \& Podsiadlowski 2007) and Yan Li et al. (e.g. Li 2000; Li \& Yang 2007) in Yunnan Observatory have made some achievements on the evolutions of single and binary stars, formation of extraordinary stars, and stellar oscillation theory.

In the field of solar physics, since the late 1980s extensive observations of solar vector magnetic fields have been made in NAOC. Lots of studies of magnetic energy accumulation and magnetic complexity in solar active regions have been carried out. Jingxiu Wang and his co-workers (Wang et al. 2007, Zhang et al. 2007) first recognized that there were large-scale source regions for the Earth-directed halo coronal mass ejections. Some global magnetic coupling has been identified by them for a few major solar activity events. In several major events trans-equatorial loops and filaments were identified to grow and erupt, which resulted in large-scale flaring.

Several important progresses have been made in the astrometric and planetary dynamics field. By the use of the 1.0/1.2m Near Earth Objects Space Telescope (NEOST), two large field of view Survey programs have been carried out and the staged achievements have been presented. Scientists in PMO have been engaged in the construction of a space debris detection system, improving notably the ability of detection and cataloging of space debrises. Astronomers in NJU studied the orbit design and control for lunar satellites, and the transfer orbit design and control for martian satellites. In the dynamics of extra-solar planetary system, astronomers in NJU provided an effective formation way of Earth-like planets, i.e. the combination between planetsimals by collisions after the formation and migration of the giant planet (Zhou et al. 2005). They also analyzed the stability of a planetary system containing $\mathrm{N}$ planets with equal mass. It was found that the essential reason of the orbital diffusion viscosity effect is the hyperbolic structure in conservative systems (Sun \& Zhou 2009).

As for the astronomical facilities, a series of telescopes have been put into operation during the past three decades, including the $2.4 \mathrm{~m}$ (Fig. 5), $2.16 \mathrm{~m}, 1.56 \mathrm{~m}, 1.2 \mathrm{~m}$ optical telescopes, the $13.7 \mathrm{~m}$ radio telescope, and the $21 \mathrm{~cm}$ array radio telescope (21CMA). Furthermore, a Chinese VLBI network (Fig. 6) has been established since 1990. There are also a series of solar telescopes, including the $35 \mathrm{~cm}$ magnetogragh at Huairou station, the $60 \mathrm{~cm}$ solar tower of Nanjing university, the radiospectrometer with high-temporal resolution, the multi-wavelength spectrograph at PMO etc.

The Large Sky Area Multi-Object Fiber Spectroscopic Telescope (LAMOST, now named Guoshoujing telescope) is one of the National Major Scientific Projects undertaken by the Chinese Academy of Sciences. The telescope was installed at the Xinglong Observing Station of NAOC in 2010. The effective aperture of LAMOST is $4 \mathrm{~m}$, with a focal plane being $1.75 \mathrm{~m}$ in diameter, corresponding to a 5 degree field of view. This may accommodate as many as 4000 optical fibers. LAMOST adopts the active optics technique both for thin mirror and segmented mirror on the Schmidt corrector, as well 


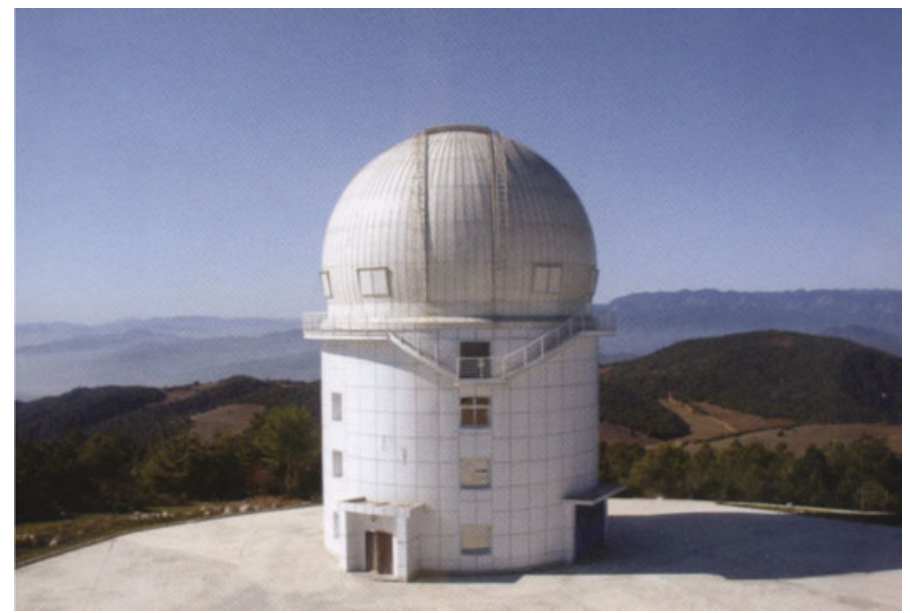

Figure 5. The $2.4 \mathrm{~m}$ telescope located at Guomeigu of Yunnan province.

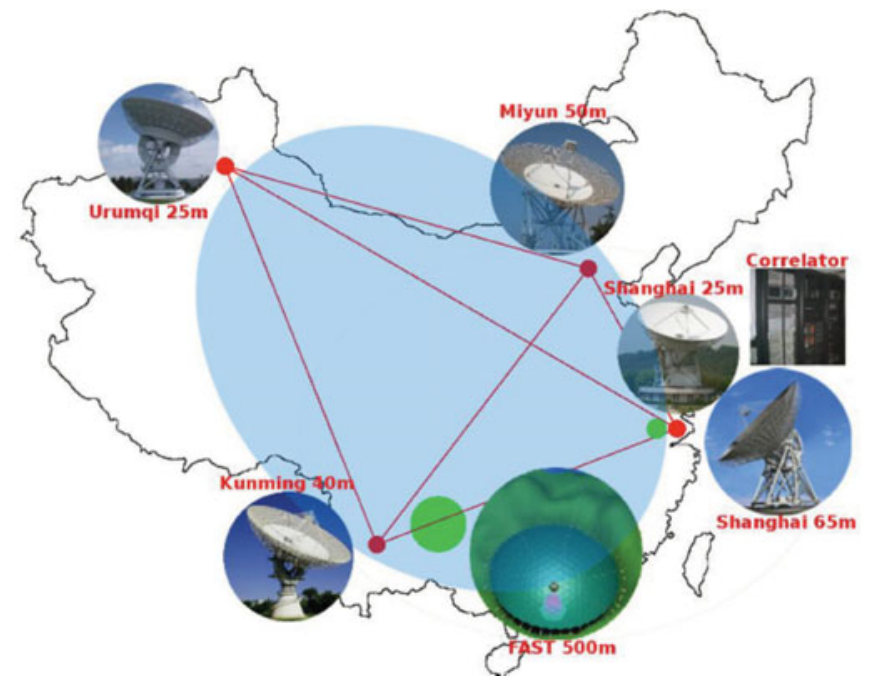

Figure 6. The Chinese VLBI network.

as the parallel controllable fiber positioning system. Thus the telescope will be the one that possesses the highest spectrum acquiring rate in the world (Cui et al. 2012). The Guoshoujing telescope will take Chinese astronomy to an advanced position in the large scale observations and the research field of optical spectra. Figure 7 gives an overview of the telescope.

The first satellite in the so-called Chang E project was launched on 2007 October 24. It obtained the first picture of the Moon on 2007 November 26. The second mission was launched in 2009 and got a full Moon high-resolution image with a resolution of $7 \mathrm{~m}$. The third mission will be carried out next year. A lunar roving vehicle will be softlanded on the Moon.

A 21 centimeter array (21CMA) has been installed at Tianshan mountains, west of China. It has 10287 antennas with $4 \mathrm{~km} \times 6 \mathrm{~km}$ arms, working in the frequency range of 50-200 MHz. It aims to probe the epoch of reionization. 


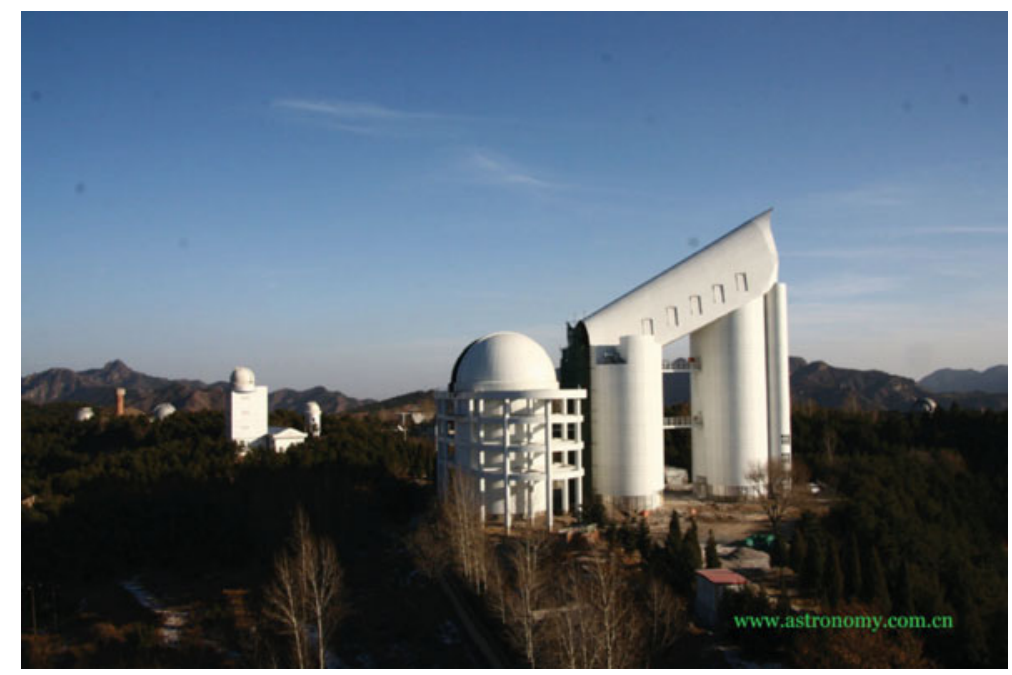

Figure 7. The Guoshoujing telescope (LAMOST) located at Xinlong, Beijing.

A $1 \mathrm{~m}$ solar vacuum telescope (NVST) and an Optical and Near-infrared Solar Eruption Tracer (ONSET) were installed at the south station of Yunnan Observatory in 2011. The station is near the Wuxian lake, $60 \mathrm{~km}$ far from Kunming, and is now the best site in China for solar observations. The $1 \mathrm{~m}$ telescope is equipped with a multi-wavelength spectrograph and several CCD cameras which can obtain solar images at different wavelengths with high resolution (Liu et al. 2011). The ONSET has three tubes and can get the solar images at white-light, $\mathrm{H} \alpha$ and HeI $10830 \AA$ simultaneously (Fang et al. 2012).

\section{Future of Astronomy in China}

The facilities under construction include the Five Hundred Meter Aperture Spherical Radio Telescope (FAST), the Chang E (Lunar mission) and Mars mission, the Hard X-ray Modulate Telescope (HXMT), the DArk Matter Particle Explorer (DAMPE), the Deep Space Solar Observatory (DSO), the Chinese Antarctic Observatory, the $65 \mathrm{~m}$ steerable radio telescope, the Chinese Spectral Radioheliogaph (CSRH), etc.

The FAST, the biggest single-dish radio telescope in the world, has an active main reflector and cable-parallel robot feed support. Its working frequencies in $\mathrm{GHz}$ are 0.3 $0.46,0.46-0.92,0.92-1.72,2.15-2.35,2.8-3.3,4.5-5.1,5.7-6.7,8.0-8.8$. The FAST project was approved by the Chinese government in 2010, and it is under construction in the southwest of China, GuiZhou provence, where an unique Karst depression can be used to put the large dish. FAST will be finished in 2016. Figure 8 depicts a sketch map of the FAST.

The HXMT aims to make hard X-ray sky (1-250 keV) survey with highest sensitivity and high precision pointed observations of high energy objects, such as black holes, AGN, SNR, neutron stars etc. Its orbit altitude is $550 \mathrm{~km}$ with an inclination of $43^{\circ}$. The HXMT will be launched in 2014-2015.

The DAMPE will search for the exact cause of cosmic ray excess, which was first observed by ATIC, and then confirmed by PAMELA FERMI and PPB-BETS experiments. Compared with other relevant space projects in the world, DAMPE has the highest energy resolution, the widest energy measurement range and the lowest background in electron/gamma detection. It will be launched in 2015 . 


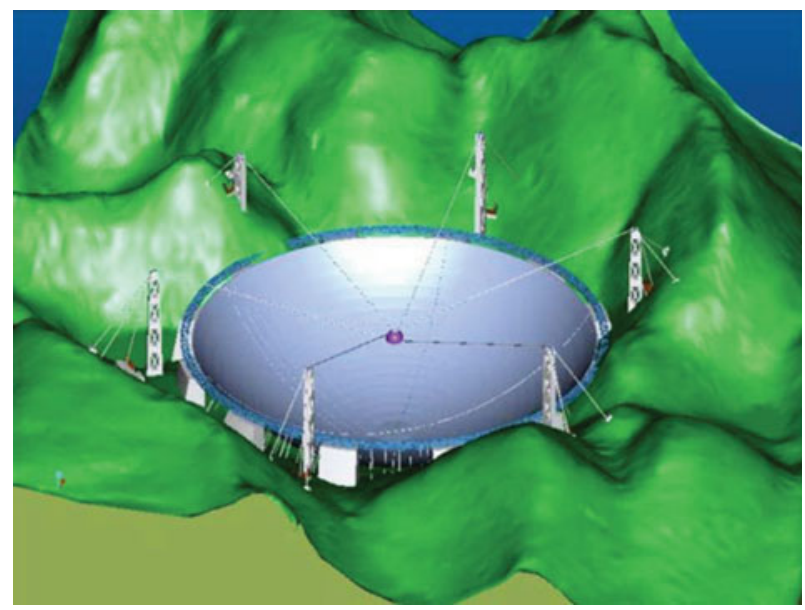

Figure 8. A sketch map of the Five Hundred Meter Aperture Spherical Radio Telescope (FAST), which is located at Guizhou province and is under construction.

The payloads of DSO include a 1m main optical telescope, an extreme ultraviolet telescope, a hard X- and $\gamma$-ray spectrometer, a Ly $\alpha$ coronagraph, a coronal imager etc. The DSO aims to observe solar magnetic field and other solar activities with high precision and high resolution. It will be launched to L1 point in about six years.

It should be mentioned that China now plans to construct a space station, on which there will be several astronomical facilities, including optical telescopes, X- and $\gamma$-ray spectrometer etc. It will certainly further strengthen much the ability of astronomical space observations in China.

The Dome A in Antarctica is possibly the best site for astronomical observations on the Earth, particularly in $\mathrm{THz}$ and infrared wavelength. The place has a very clear sky and low wind speed, with low thickness of ground layer and very good transmission from 150 to $800 \mu \mathrm{m}$. After a Chinese expedition team reached Dome A in 2005 January, Chinese astronomers followed on with international collaborations. Recently the Dome A observatory project is approved by the Chinese government. A $2.5 \mathrm{~m}$ optical/infrared telescope and a $5 \mathrm{~m} \mathrm{THz}$ telescope will be installed at the observatory, aiming at studying dark energy and dark matter, exo-planets, time-domain subjects, formation and evolution of stars and galaxies etc.

A $65 \mathrm{~m}$ fully steerable radio telescope is also under construction at Shanghai observatory. It will be working at the frequencies of $\mathrm{L}(1.6 \mathrm{GHz}), \mathrm{S} / \mathrm{X}(2.3 / 8.4 \mathrm{GHz}), \mathrm{C}(5 \mathrm{GHz})$, $\mathrm{Ku}(15 \mathrm{GHz}), \mathrm{K}(22 \mathrm{GHz}), \mathrm{Ka}(32 \mathrm{GHz})$, and $\mathrm{Q}(43 \mathrm{GHz})$. It will be used in precise astrometry and astrophysics, such as observations of high-resolution VLBI, maser sources, molecular spectral line survey, radio stars etc. The telescope will be put into operation in 2015.

Another big project is the Chinese Spectral Radioheliogaph (CSRH), which has been under construction since 2009 at Zhengxiang Baiqi, Inner Mongolia. It will be working in the frequency range of $0.4-15 \mathrm{GHz}$, with 100 parabolic antennas, and achieve 1.3-50 arcsecond spatial resolution and better than 100ms temporal resolution. The CSRH will be put into operation in 2014 .

Besides, some projects are under discuss and review. These include a $110 \mathrm{~m}$ radio telescope, 20-30m optical/infrared Telescope, south LAMOST, large ground-based solar observatory (Chinese Giant Solar Telescope (CGST) and coronagraphs), new generation 
Chinese VLBI network, X-ray Timing and Polarization Mission (XTP), advanced spaceborn solar observatory etc.

It should be mentioned that an extensive site survey both for the night- and day-time astronomy in the western China has been started since the beginning of this century. If the best site can be found, then China will build new large optical telescopes for the solar and stellar observations respectively.

\section{Conclusions}

Through out the ancient history, Chinese astronomers had made tremendous achievements. However, in the past several centuries, Chinese astronomy research has been much delayed behind comparing to the western countries. Since the beginning of the People's Republic of China, and in particular, after the so-called great cultural revolution, Chinese astronomy has been developed quickly. Benefiting from the fast development of Chinese economy, the research of astronomy in China has made remarkable progress in the recent years. The numbers of astronomers and the graduate students have been greatly increased. The budget for astronomy research is ten times larger than that ten years ago. Recently, Chinese astronomers have obtained many important results. A series of astronomical facilities have been put into operation, including the Guoshoujing telescope, which possesses the highest spectrum acquiring rate in the world. Particularly, China has many exciting projects to build advanced both ground-based and space-born telescopes. If these plans can be successfully put in practice, Chinese astronomy will enter into a new era! The future will be highly luciferous.

We understand that at present there is still a big gap between Chinese astronomy and that in advanced countries. We have a lot of catch-up to do and we believe that the international cooperation is essential during this journey. All suggestions and help from astronomers and friends over the world are highly welcome!

\section{Acknowledgements}

Thanks to the organizers for providing the opportunity to show Chinese astronomy from the past to the future. Thanks to my friends and colleagues for the use of their data and publications that help me prepare this paper. This work received support from the grant of the 973 project 2011CB811402 of China, and the National Natural Science Foundation of China (NSFC) under the grant numbers 10878002, 10610099, 10933003 and 10673004 .

\section{References}

Chang, J., Adams, J. H., Ahn, H. S., et al. 2008, Nature, 456, 362

Cui, X. Q., Zhao, Y. H., \& Chu, Y. Q., et al. 2012, RAA, 12, 1197

Dai, Z. G., Liang, E. W., \& Xu, D. 2004, ApJ, 612, 101

Fang, C., Chen, P. F., Ding, M. D., et al. 2012, in proceeding of the 4th France-China meeting on solar physics, eds. M. Faurobert, C. Fang, T. Corbard, EAS Publication Series, Vol.55, 349

Han, J. L., Manchester, R. N., \& Qiao, G. J. 1999, MNRAS, 306, 371

Han, J. L., Manchester, R. N., Lyne, A. G., Qiao, G. J., \& van Straten, W. 2006, ApJ, 642, 868

Han, Z. \& Podsiadlowski, Ph 2008, IASS, 252, 349

Hou, L. G., Han, J. L., \& Shi, W. B. 2009, A $\& A$, 499, 473

Jing, Y. P. \& Suto, Y. 2002, ApJ, 574, 538

LI, Y. 2000, ApJ, 538, 346

Li, Y. \& Yang, J. Y., 2007, MNRAS. 375, 388 
Liu, Z. \& Xu, J. 2011, in First Asia-Pacific Solar Physics Meeting, ASI Conference Series, eds.

A. R. Choudhuri \& D. Banerjee, Vol. 2, 9

Sun X. 2011, IAU Symp. 260, 98

Wang, J. X., Zhang, Y. Z., Zhou, G. P., Harra, L. K., Williams, D. R., \& Jiang, Y. C. 2007, Solar Phys., 244, 75

Xu, Y., Reid, M. J., Zheng, X. W., \& Menten, K. M. 2006, Science, 311, 54

Xu, Y., Reid, M. J., Menten, K. M., Brunthaler, A., Zheng, X. W., \& Moscadelli, L. 2009, ApJ, 693,413

Xu Z. T. \& Jiang Y. T. 1986, Sunspots and Human, Tianjin Science and Technic Publ.

Zhang, Y. Z., Wang, J. X., Attrill, G. D. R., Harra, L. K., Yang, Z. L., \& He, X. T. 2007, Solar Phys., 241, 329

Zhao, D., Jing, Y. P., Mo, H. J., \& Borner, G. 2009, ApJ, 707, 354

Zhao, G. \& Gehren, T. 2000, A\&\&A, 362, 1077

Zhou, J. L., Aarseth, S. J., Lin, D. N. C., \& Nagasawa, M. 2005, ApJ, 631, L85

Sun, Y. S. \& Zhou, L. Y., 2009, Celest Mech Dyn Astr, 103, 119 